\title{
ETDRS Grading of Diabetic Retinopathy: Still the Gold Standard?
}

\author{
Sharon D. Solomon Morton F. Goldberg \\ Wilmer Eye Institute, Baltimore, MD, USA
}

\section{Keywords}

ETDRS Grading · Diabetic retinopathy · Clinically significant macular edema · Intraretinal microvascular abnormalities

\begin{abstract}
This article aims to provide a narrative history of the evolution, modification, and legacy of the Early Treatment Diabetic Retinopathy Study classification system.
\end{abstract}

(c) 2019 S. Karger AG, Basel

Some 50 years ago, on September 29, 1968, an international consortium of leaders in ophthalmology, internal medicine, and neurosurgery gathered at Airlie House, Warrenton, VA, to address the growing health concern around diabetic retinopathy as a leading cause of blindness (Fig. 1). The goals of the symposium were threefold: to promote dialogue among clinical investigators and practitioners focused on the treatment of diabetic retinopathy, to assess currently available data regarding the efficacy of pituitary ablation and photocoagulation in the management of diabetic retinopathy, and to establish an

\section{KARGER}

(๑) 2019 S. Karger AG, Basel

E-Mail karger@karger.com

www.karger.com/ore objective classification of diabetic retinopathy that would facilitate comparisons of the natural course of the disease and the response to various treatments at different centers [1]. This article aims to provide a narrative history of the evolution, modification, and legacy of this classification system for diabetic retinopathy.

Realizing, first, that a preliminary, standard classification of diabetic retinopathy with which data could be analyzed and compared amongst investigators at this US Public Health Service-sponsored symposium was essential, a group of investigators (Drs. M.D. Davis, S.L. Fine, M.F. Goldberg, J.W. McMeel, E.W.D. Norton, E. Okun, and P. Wetzig) met at the O'Hare Inn, Des Plaines, IL, on June 29, 1968, in advance of the Airlie House Symposium. What they developed, The O'Hare Classification for diabetic retinopathy, enabled those participating in the subsequent Airlie House Symposium to prepare cases and to present data using a basic system of categorization involving four characteristics of diabetic retinopathy: background retinopathy, vitreous hemorrhage, new vessels, and fibrous proliferation extending into the vitreous cavity [1]. Despite its limitations, the O'Hare Classification was valuable as a framework for the main symposium that allowed comparison of results obtained from various 


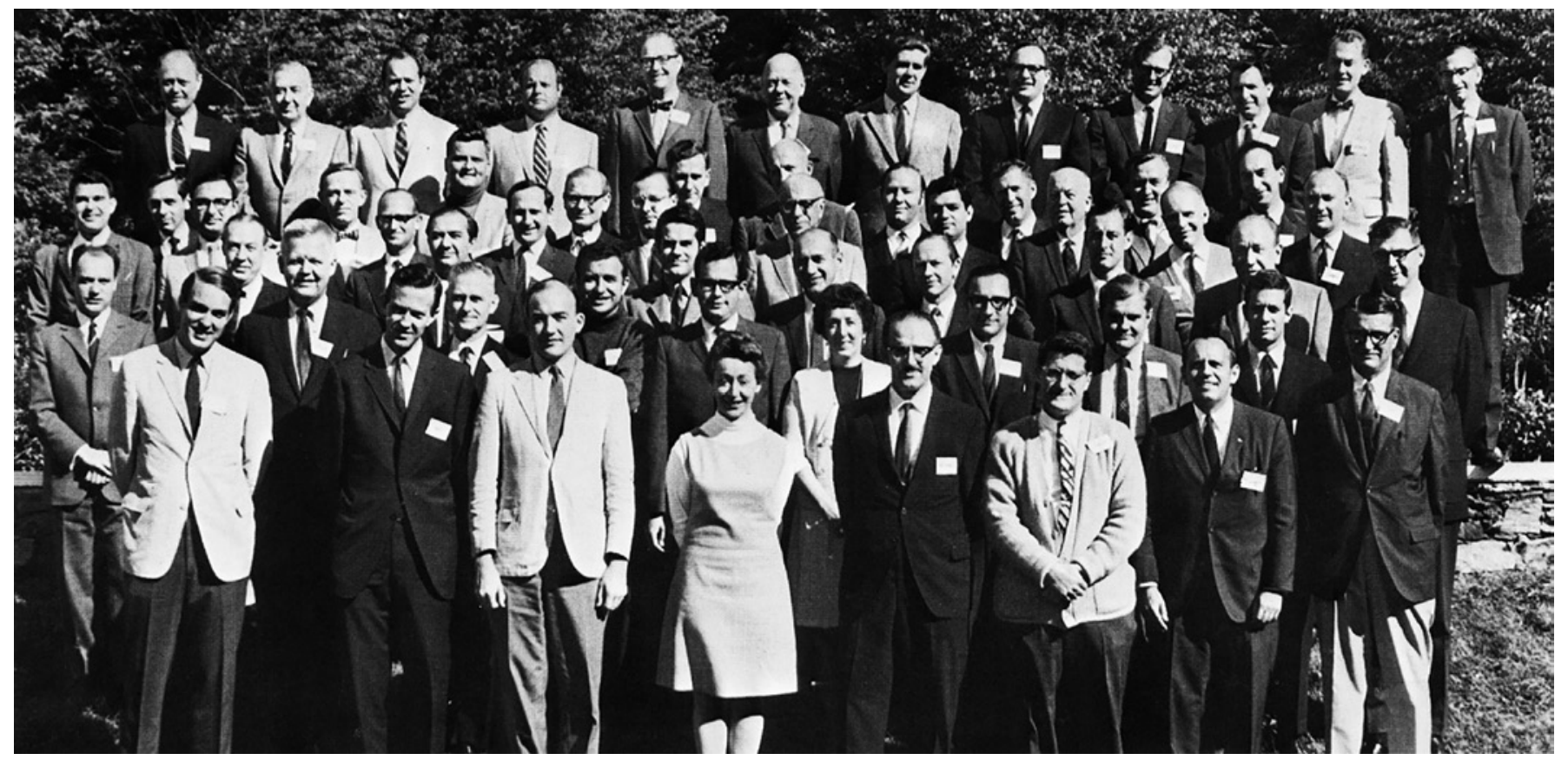

Fig. 1. Participants of the Airlie House Symposium on the Treatment of Diabetic Retinopathy, September 29 to October 1, 1968. Row 1 (front): S.L. Fine, M.D. Davis, N. Oakley, E. Kohner, M. Balodimos, H. Spalter, J. Linfoot, T. Duane. Row 2: A. Wessing, R. Kjellberg, W. Sweet, N. Zervas, E. Finkelstein, E. Hallworth, L. Jagerman, E.W.D. Norton, H. Lester. Row 3: B. Ray, J. Hardy, A. Panisset, E. Okun, B. Straatsma, G. Cleasby, O. Pearson, J.W. Mc-
Meel. Row 4: F. Myers, M. Goldberg, H.-W. Larsen, R. Blach, T.R. Fraser, W. van Heuven, R. Feinberg, K. Gabbay, G. McDonald, J. Dobree, R. Schimek. Row 5: R. Packman, R. Bradley, P. Jahnke, F. Caird, P. Thornfeldt, C. Mortimer, G. Harris, K. Lundbaek, H. Keen. Row 6: P. Wetzig, W. Beetham, W. Peretz, J. Glaser, M. Rubin, J. Ferree, Sv. Simonsen, N. Roth, H.C. Zweng, L. Aiello, E. Greenberg, G, Joplin. therapeutic interventions at several different medical centers.

Empowered with this common language from the O'Hare Classification, the Airlie House committee on classification developed a more comprehensive system to critically describe diabetic retinopathy and to evaluate the effect of treatment modalities. The Airlie House Classification of diabetic retinopathy qualitatively and quantitatively described the status of diabetic retinopathy and employed fundus photography to permanently record the locations of these lesions in certain predesignated areas of the fundus (Fig. 2). That individual photographs could be graded consistently by experienced, independent assessors was recognized by the committee as an essential feature of any classification scheme, especially one that would establish the natural course of diabetic retinopathy by statistical analysis of serial examinations as well as objectively evaluate the effects of therapeutic interventions. Separate color photographic standards were established for hemorrhages, hard exudates, venous abnormalities, and neovascular and fibrous proliferation within one disc diameter of the disc. Photographic fields were chosen

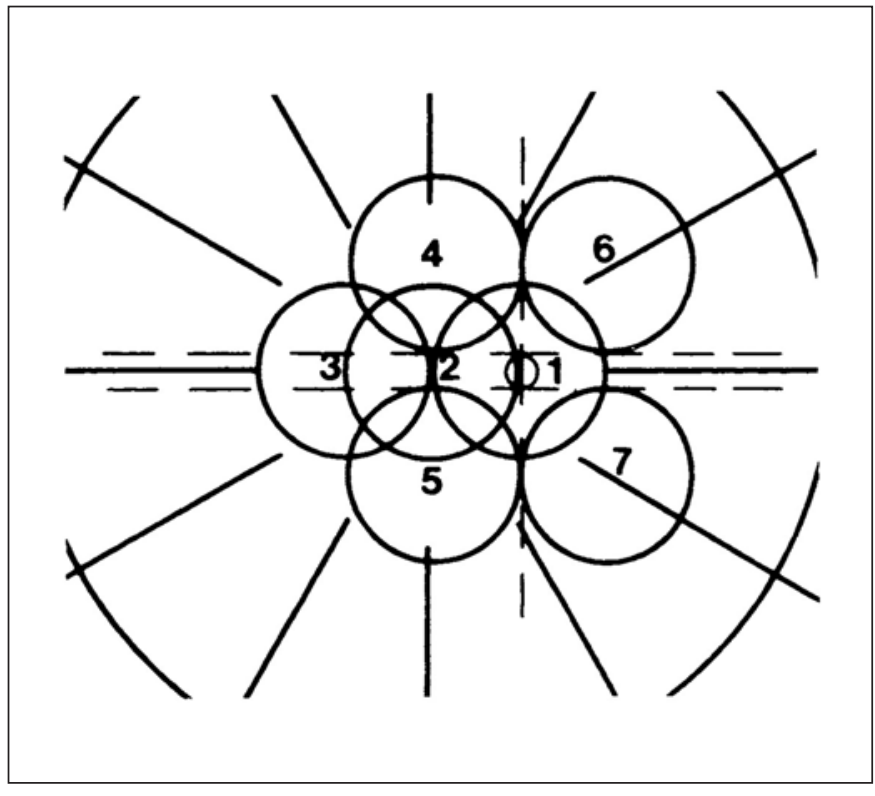

Fig. 2. Seven standard fields of the modified Airlie House classification of diabetic retinopathy. In this right eye, field 1 is centered on the optic nerve. Field 2 is centered on the macula, and field 3 is temporal to the macula. 

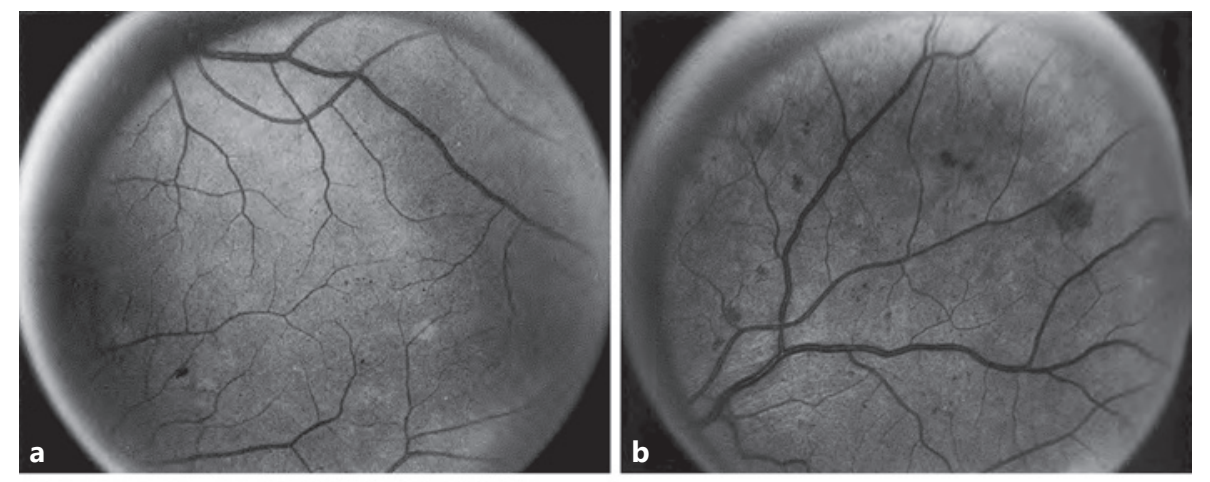

Fig. 3. a Standard photograph 1 , showing a mild degree of microaneurysms and hemorrhages. b Standard photograph 2A, showing an intermediate degree of microaneurysms and hemorrhages. c Standard photograph 2B, showing a severe degree of microaneurysms and hemorrhages.

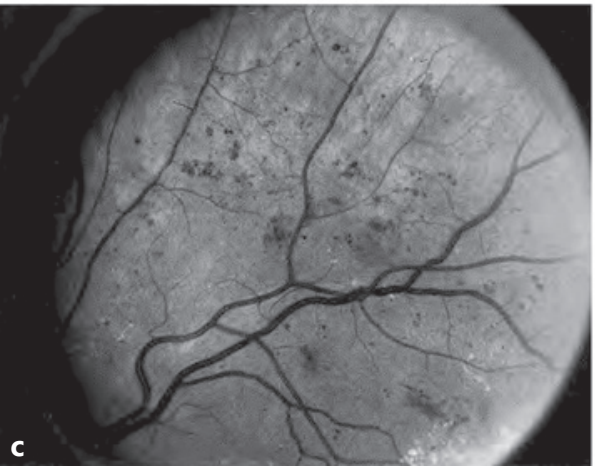

based on their importance for visual function, such as the disc and macula (Fig. 2), and for the frequency of their involvement in early diabetic retinopathy, such as the area temporal to the macula (Fig. 3). The committee's confidence that the widespread acceptance of the Airlie House Classification of diabetic retinopathy would encourage and facilitate its prospective use in future cooperative studies was validated at the conclusion of the symposium by requests for and distribution of sets of standard photographs to the United Kingdom, the United States, Switzerland, Germany, Australia, Italy, South Africa, Malta, Greece, and Brazil.

Indeed, a few years later in 1971, the Airlie House Classification of diabetic retinopathy was modified for use in the first landmark clinical trial, the Diabetic Retinopathy Study (DRS), which demonstrated a significant reduction in the rates of severe vision loss in eyes treated with panretinal photocoagulation compared to untreated control eyes [2]. The seven standard photographic fields and color stereoscopic fundus photographs were used to define high-risk proliferative diabetic retinopathy (HRPDR) as either mild neovascularization ( $1 / 4$ to $1 / 3$ disc area) of the disc (NVD) with vitreous hemorrhage, moderate to severe NVD with or without vitreous hemorrhage, or moderate neovascularization (1/2 disc area) elsewhere with vitreous hemorrhage. Because of this universally recog- nized classification system, in combination with the use of color stereoscopic fundus photographs, eyes with highrisk features could easily and consistently be identified and targeted for treatment by ophthalmologists around the globe with the expectation that results would mirror those obtained in this randomized, prospective trial.

The modified Airlie House classification of diabetic retinopathy used in the DRS was further developed for use in the Early Treatment Diabetic Retinopathy Study (ETDRS), a randomized, prospective study that evaluated the efficacy of laser treatment for macular edema. The revisions to the classification system added steps to the grading scale for some fundus features and separated other fundus features that had previously been combined. For instance, additional steps were added to the existing grading scale for hard exudates, soft exudates, arteriovenous nicking, retinal elevation, and vitreous hemorrhage. Three abnormalities that were combined in the DRS grading protocol were assessed separately in the ETDRS: venous beading, venous narrowing, and venous loops or reduplication. In addition, several important fundus features were added that had not previously been graded, such as microaneurysms and intraretinal microvascular abnormalities (IRMA). Most notably, however, the ETDRS grading criteria expanded the description and classification of diabetic macular edema, and this second 
Fig. 4. a Standard photograph 6A, showing less severe degree of venous beading. b Standard photograph 6B, showing more severe degree of venous beading.
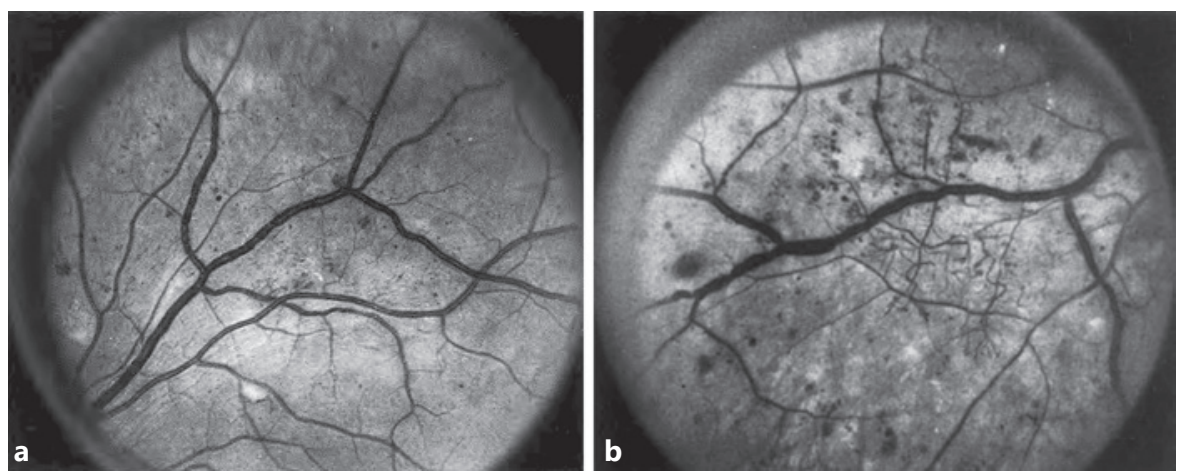

Fig. 5. a Standard photograph $8 \mathrm{~A}$, showing less severe degree of IRMA (see insets). $\mathbf{b}$ Standard photograph 8B, showing more severe degree of IRMA (see inset).
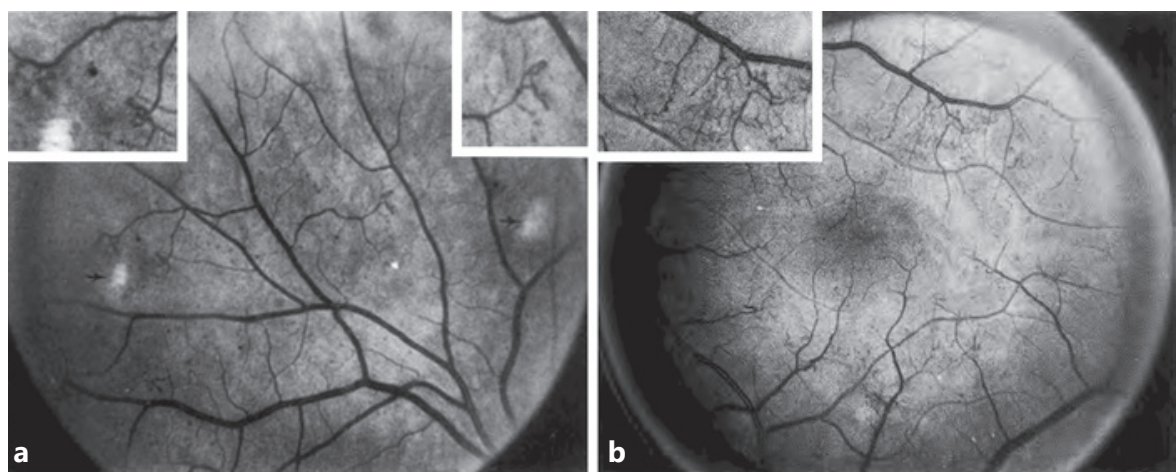

landmark clinical trial showed that the rate of moderate vision loss was reduced by at least $50 \%$ in laser-treated eyes with clinically significant macular edema (CSME) compared to untreated eyes [3].

In the ETDRS, a microaneurysm was defined as a red spot $125 \mu \mathrm{m}$ in its longest dimension with sharp margins. It was distinguished from a hemorrhage, which was defined as a red spot with irregular margins and a dimension that exceeded $125 \mu \mathrm{m}$. Using the area of retina covered by microaneurysms and/or hemorrhages for comparison with standardized fundus photographs, a grading scale emerged for description of non-proliferative diabetic retinopathy (NPDR). The grading scale was as follows: grade $0=$ no microaneurysms or hemorrhages; grade $1=$ questionable microaneurysms or hemorrhages; grade $2=$ definite microaneurysms or hemorrhages less than standard photograph 1; grade 3 = microaneurysms or hemorrhages greater than or equal to standard photograph 1 but less than standard photograph $2 \mathrm{~A}$; grade $4=$ microaneurysms or hemorrhages greater than or equal to standard photograph $2 \mathrm{~A}$ but less than standard photograph $2 \mathrm{~B}$; grade $5=$ microaneurysms or hemorrhages greater than or equal to standard photograph 2B (Fig. 3) [3].
Localized increases in venous caliber, or venous beading, were graded in the ETDRS based on the total length of vein involved and on the severity of beading, using standard photographs $6 \mathrm{~A}$ and $6 \mathrm{~B}$ (Fig. 4). In fields 3-7, grade 0 = no beading; grade $1=$ questionable beading; grade 2 = definite beading but less than standard photograph $6 \mathrm{~A}$; grade $3=$ beading greater than or equal to standard photograph 6A but less than standard photograph $6 \mathrm{~B}$; and grade $4=$ beading equal to or greater than standard photograph $6 \mathrm{~B}[3]$.

IRMA in the ETDRS was characterized as tortuous intraretinal vascular segments that varied in width from barely visible to approximately one-fourth the diameter of a major vein at the disc margin or $31 \mu \mathrm{m}$. Grading was again based on the area of the retina covered by IRMA using standard photographs $8 \mathrm{~A}$ and $8 \mathrm{~B}$ for comparison (Fig. 5). As with other fundus features that characterized the severity of NPDR, the scale was precise and reproducible with grade $0=$ no IRMA; grade $1=$ questionable IRMA; grade 2 = definite IRMA but less than standard photograph $8 \mathrm{~A}$; grade $3=$ IRMA greater than or equal to standard photograph $8 \mathrm{~A}$ but less than standard photograph $8 \mathrm{~B}$; and grade $4=$ IRMA greater than or equal to standard photograph 8B [3]. 
In describing CSME, the single DRS question regarding macular edema was extended in the ETDRS with assessments of location, extent, and severity of retinal thickening in the posterior pole. CSME was defined as either retinal edema located at or within $500 \mu \mathrm{m}$ of the center of the macula, hard exudates at or within $500 \mu \mathrm{m}$ of the foveal center if associated with thickening of adjacent retina, or as a zone of thickening larger than 1 disc area if located within 1 disc diameter of the center of the macula [3]. As one of the only fundus features graded in field 2 in the ETDRS, the presence of CSME was the main criterion used to mandate treatment in eyes assigned to early laser photocoagulation in this randomized clinical trial.

The longevity of the ETDRS classification of diabetic retinopathy and its relevance in both clinical and research settings over the last half century lie primarily in its reproducibility and applicability. In traditional assessments of the level of diabetic retinopathy, baseline color fundus photographs would be graded independently by two graders. The initial field by field gradings of each member of the grader pair would be entered into a computer for comparison [3]. If disagreements on the grading scale were separated by one step, they would be averaged. If there were grader disagreements of two steps or more, the characteristics of the fields in question would be graded, again, by each grader. If the two or more step disagreement was not resolved, a more senior adjudicator, who remained masked to the original grades, would decide the final grades for the involved fields. In the ETDRS, the reproducibility between graders was assessed by calculating percentages of agreement and kappa statistics for duplicate gradings of baseline color stereoscopic fundus photographs. Reproducibility was found to be very reliable for most characteristics. What started as a classification system accepted by academic reading centers (handling large volumes of photographic data for clinical trials outcomes) also became applicable in the everyday clinical setting. Retina specialists were able to grade the severity of patients' diabetic retinopathy in order to establish guidelines for follow-up intervals in-office and to assess criteria that indicated the need for intervention with photocoagulation for CSME or for HRPDR. For the last halfcentury, the ETDRS classification of diabetic retinopathy has remained the universal standard by which all useful fundus findings of affected patients are described.

However, with randomized clinical trials demonstrating that there may be serendipitous regression of background diabetic retinopathy when center-involved diabetic macular edema (CIDME) is treated with intravitreal anti-vascular endothelial growth factor (anti-VEGF) agents, the ETDRS classification of diabetic retinopathy may be less clinically relevant $[4,5]$. Not only do the antipermeability properties of these anti-VEGF agents promote anatomic resolution of CIDME, favorably affecting visual acuity outcomes, but also their anti-angiogenesis properties have been shown to retard or even reverse the progression of many features of diabetic retinopathy. Indeed, the mere rebranding of "CSME" (a diagnosis traditionally made with a fundus contact lens during slit-lamp biomicroscopy) with "CIDME" (a diagnosis made with optical coherence tomography or OCT) reflects a changing of the guard, as ETDRS classifications are modified.

The first large-scale, randomized clinical trial to show improved visual acuity outcomes and anatomic outcomes in patients with CIDME randomized to intravitreal therapy with ranibizumab and deferred laser, compared to laser alone, established anti-VEGF agents as the first line therapy for CIDME [5]. Since that time, extended followup of these patients through 5 years has demonstrated improvement in severity of diabetic retinopathy [6]. In this trial, assessment of diabetic retinopathy severity was performed by masked graders in a central reading center, using standard 7-field color fundus photographs obtained at baseline, 1 year, and annually between years 3 and 5. Changes in diabetic retinopathy severity were assessed for both eyes with NPDR and with PDR. The NPDR group was further subdivided into ETDRS classifications of moderate or better NPDR, moderate-severe NPDR, or severe NPDR. The study reported that the proportion of eyes with improvement in diabetic retinopathy severity was similar over time and between the NPDR and PDR subgroups. At the 5-year outcomes visit, $32 \%$ of the NPDR group and $23 \%$ of the PDR group improved with respect to diabetic retinopathy severity [6]. Within the NPDR subgroups, $15 \%$ of the moderate or better NPDR group and $42 \%$ of the moderate-severe NPDR group showed improvement in diabetic retinopathy severity [6]. Importantly, eyes with NPDR receiving anti-VEGF therapy were also observed to be less likely to worsen compared to eyes with PDR, with a 5-year cumulative probability of worsening of $18 \%$ for the NPDR group and $31 \%$ for the PDR group [6].

The clinical relevance of the ETDRS classification of diabetic retinopathy has been challenged not only by therapies that cause disease regression but also by technological advances in screening and image acquisition of the fundus features of diabetic retinopathy. With the advent of ultrawide field imaging, clinicians are now able to assess and document peripheral changes in the fundus features and retinal circulation of diabetic patients with a 
modality that is less cumbersome to obtain than the traditional standard 7-field color fundus photographs. In combination with software that allows for automated algorithms that utilize widefield imaging to detect and grade diabetic retinopathy with high sensitivity [7], screening as well as accurate staging of diabetic retinopathy may become accessible and reproducible outside of the ophthalmologist's office.

For decades, the natural evolution of diabetic retinopathy has been well described and classified by the ETDRS retinopathy scale, based on assessment of fundus features photographically documented and compared with standard color fundus photographs. Disease progression in the eyes of affected patients was observed to be orderly. In addition, progression through the various steps of the ETDRS scale could be interpreted by the treating physician as an escalated risk of vision-threatening retinopathy. Now that anti-VEGF agents have been shown to re- verse the progression of diabetic retinopathy, is it time to retire the ETDRS classification of diabetic retinopathy? This question becomes more complex when the limitation of assessing diabetic retinopathy severity within the traditional ETDRS 7-fields is removed, and fundus findings in diabetic patients are assessed with ultrawide-field (UWF) imaging. UWF imaging has already demonstrated the potential to identify a subset of patients at increased risk of progression of diabetic retinopathy severity that cannot be assessed with traditional ETDRS 7-field imaging [8]. It may well be time for the next generation of international leaders in ophthalmology to convene to systematically address these issues.

\section{Disclosure Statement}

Neither Dr. Sharon D. Solomon nor Dr. Morton F. Goldberg has any relevant financial conflicts to disclose.

\section{References}

1 Goldberg MF, Fine SL. Symposium on the Treatment of Diabetic Retinopathy. Arlington: US Department of Health, Education, and Welfare; 1968. Public Health Service Publication No. 1890.

2 The Diabetic Retinopathy Study Research Group. A Modification of the Airlie House Classification of Diabetic Retinopathy. DRS report \#7. Invest Ophthalmol Vis Sci. 1981;21: $210-26$.

3 Early Treatment Diabetic Retinopathy Study Research Group. Grading Diabetic Retinopathy from Stereoscopic Color Fundus Photographs-An Extension of the Modified Airlie House Classification. ETDRS Report Number 10. Ophthalmology. 1991 May;98 (5):786806.
4 Brown DM, Nguyen QD, Marcus DM, Boyer DS, Patel S, Feiner L, et al.; RIDE and RISE Research Group. Long-term outcomes of ranibizumab therapy for diabetic macular edema: the 36-month results from two phase III trials: RISE and RIDE. Ophthalmology. 2013 Oct;120(10):2013-22.

5 Elman MJ, Aiello LP, Beck RW, Bressler NM, Bressler SB, Edwards AR, et al.; Diabetic Retinopathy Clinical Research Network. Randomized trial evaluating ranibizumab plus prompt or deferred laser or triamcinolone plus prompt laser for diabetic macular edema. Ophthalmology. 2010 Jun;117(6):1064-1077. e35.
6 Bressler SB, Odia I, Glassman AR, Danis RP, Grover S, Hampton GR, et al. Changes in diabetic retinopathy severity when treating diabetic macular edema with ranibizumab: DRCR.net Protocol I 5-Year Report. Retina. 2018 Oct;38(10):1896-904.

7 Wang K, Jayadev C, Nittala MG, Velaga SB, Ramachandra CA, Bhaskaranand $\mathrm{M}$, et al. Automated detection of diabetic retinopathy lesions on ultrawidefield pseudocolour images. Acta Ophthalmol. 2018 Mar;96(2):e168-73.

8 Aiello LP, Odia I, Glassman AR, Melia M, Jampol LM, Bressler NM, et al.; Diabetic Retinopathy Clinical Research Network. Comparison of Early Treatment Diabetic Retinopathy Study Standard 7-Field Imaging With Ultrawide-Field Imaging for Determining Severity of Diabetic Retinopathy. JAMA Ophthalmol. 2019 Jan;137(1):65-73. 